Dieses Dokument ist eine Zweitveröffentlichung (Verlagsversion) / This is a self-archiving document (published version):

Adrian Ehrenhofer, Thomas Wallmersperger

Active hydrogel composite membranes for the analysis of cell size distributions

Erstveröffentlichung in / First published in:

SPIE Smart Structures + Nondestructive Evaluation. Denver, 3. - 7.03.2019. SPIE Digital Library, Art. Nr. 1096815 [Zugriff am: 25.03.2021]. ISBN 978-1-51062-5-921.

DOI: https://doi.org/10.1117/12.2513199

Diese Version ist verfügbar / This version is available on:

https://nbn-resolving.org/urn:nbn:de:bsz:14-qucosa2-742377 


\section{Active hydrogel composite membranes for the analysis of cell size distributions}

Ehrenhofer, Adrian, Wallmersperger, Thomas

Adrian Ehrenhofer, Thomas Wallmersperger, "Active hydrogel composite membranes for the analysis of cell size distributions," Proc. SPIE 10968, Behavior and Mechanics of Multifunctional Materials XIII, 1096815 (29 March 2019); doi: 10.1117/12.2513199

Event: SPIE Smart Structures + Nondestructive Evaluation, 2019, Denver, Colorado, United States 


\title{
Active hydrogel composite membranes for the analysis of cell size distributions
}

\author{
Adrian Ehrenhofer ${ }^{\mathrm{a}}$ and Thomas Wallmersperger ${ }^{\mathrm{a}}$ \\ anstitut für Festkörpermechanik, Technische Universität Dresden, 01062 Dresden
}

\begin{abstract}
Active membranes with switchable pores that are based on hydrogels can be used to measure the cell size distribution in blood samples. The system investigated in the present research is based on a polyethylene terephthalate (PET) membrane that is surface polymerized with poly(N-isopropyl acrylamide) (PNiPAAm) to form active pores of arbitrary geometry. The PET membrane provides the functionality of a backbone for mechanical rigidity, while the soft PNiPAAm hydrogel forms the active pores. Modeling and simulation of the active hydrogel behavior proved to adequately predict the opening and closing of the pores under application of an activating stimulus, e.g. temperature. The applied model is called Temperature-Expansion-Model and uses the analogy of thermal expansion to model the volume swelling of hydrogels. The Normalized Extended Temperature-Expansion-Model can englobe arbitrary hydrogels and large geometric displacements. Studies of pore opening - performed by using commercial finite element tools - show good agreement of the experimentally measured shape change of active pores. Based on these studies, the particulate fluid flow through the switchable pores is analyzed. Through application of a membrane process, i.e. a given variation of applied pressure and switching stimulus for the hydrogel, the size profile of the blocking particles can be measured directly using the flux difference under constant pressure. This allows the measurement of the cell size distribution in blood samples, e.g. to detect circulating tumor cells or anomalies in the distribution that hint to anemia.
\end{abstract}

Keywords: Permeation control membranes, Hydrogels, Modeling and Simulation, Microfluidics, Particle Separation

\section{INTRODUCTION}

Hydrogel-layered composite membranes have a great potential for measurements in particle-laden microfluidic flows. In our previous work, ${ }^{1}$ we presented the concept of active membranes which are composed of a backbone PET membrane and are surface polymerized with PNiPAAm, see Figure 1. In the subsequent works on this topic, we investigated the pore forms, ${ }^{2}$ microfluidic flow through the system ${ }^{3}$ and the mechanical behavior. ${ }^{4}$ The goal of the current work is now to describe a measurement process for particle size distributions with this system in order to prove that it is suitable for this task.

Hydrogels are a class of active materials that are characterized by reversible swelling of hydrophilic polymers in water. ${ }^{5}$ This swelling can be due to different mechanisms, such as polymer chain interactions or hydrophilic interactions with the surrounding medium. ${ }^{6}$ Modeling and simulation of hydrogels can be performed on the basis of continuum mechanics e.g. by applying the Theory of Porous Media ${ }^{7-9}$ or the multifield theory. ${ }^{10}$

State of the art characterization of biological cells mostly comprises label-based sorting methods like fluorescent activated cell sorting (FACS) or magnetic activated cell sorting (MACS). ${ }^{11}$ Sorting of cells can also be performed based on the mechanical properties of cells. ${ }^{12}$ Other concepts for switchable membranes use the hydrogel's change of porosity. ${ }^{13}$

In the current work, in section 2 we briefly describe the behavior of the active membrane system. In the following, we discuss the active behavior and the resulting flow and filtration of the membrane. Then, we describe

Further author information:

Adrian Ehrenhofer: E-mail: Adrian.Ehrenhofer@tu-dresden.de

Thomas Wallmersperger: E-mail: Thomas.Wallmersperger@tu-dresden.de

Behavior and Mechanics of Multifunctional Materials XIII, edited by Hani E. Naguib, Proc. of SPIE Vol. 10968, 1096815 - (c) 2019 SPIE · CCC code: 0277-786X/19/\$18 · doi: 10.1117/12.2513199 


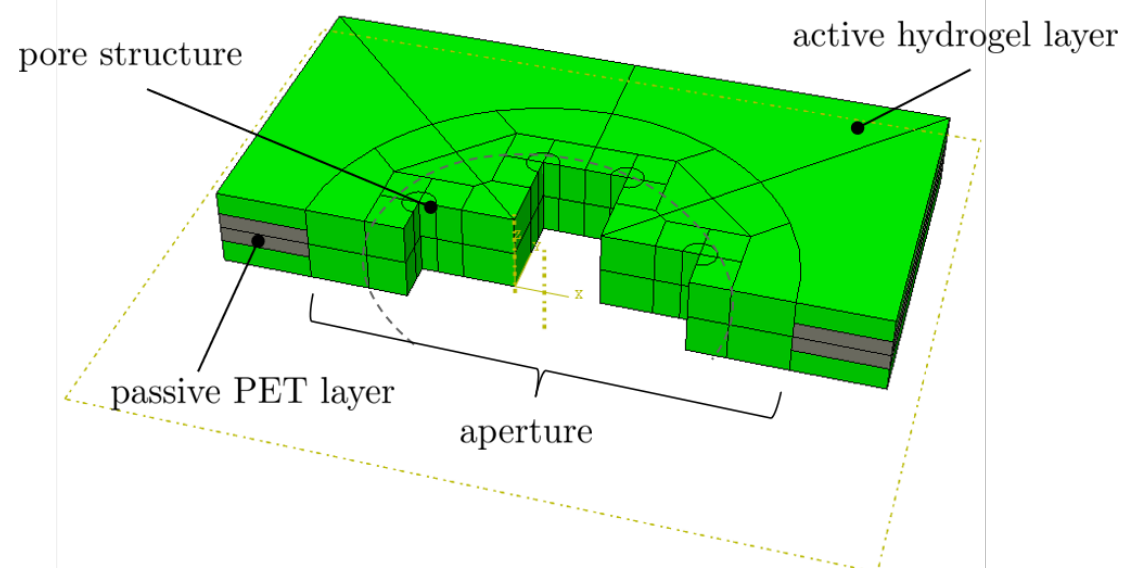

Figure 1: Membrane setup with passive PET layer, active hydrogel layer, aperture and pore structure.

the measurement process for cell size profiles and present a circuit model which is suitable to describe the process. In section 3, we show the simulation of the measurement process to prove that the active membrane system is indeed capable of measuring cell size profiles.

\section{BEHAVIOR OF THE ACTIVE MEMBRANE SYSTEM}

The main aspect of the presented system is the possibility to control pore diameters, see section 2.1. From this behavior follows (i) the control of flow and with the description of discrete blocking follows (ii) the switchability of particle permeation, see section 2.2. In the next step, in section 2.3 we explore a cell size measurement process. In order to describe this process, we derive a circuit model in section 2.4 which is applied in section 3 to obtain deeper insights into the measurement results and their interpretation.

\subsection{Controllable opening of membrane pores}

When the PNiPAAm hydrogel is subjected to an increasing temperature, it responds with deswelling due to Lower Critical Solution Temperature (LCST) behavior, see Figure 2a. Since the hydrogel is fixed at the backbone membrane, this leads to an opening of the membrane with higher temperatures, see figure $2 \mathrm{~b}$.

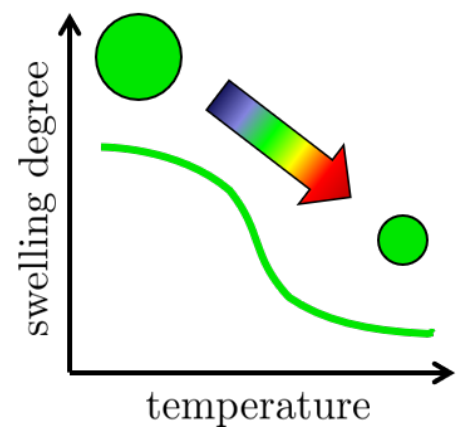

(a)

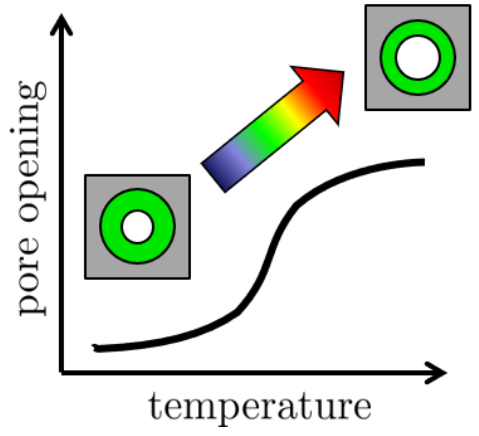

(b)

Figure 2: (a) Swelling curve of a PNiPAAm sample under free swelling conditions. This behavior translates to (b) pore opening when being under circular constraint.

Previous works have shown that the opening and closing of pores with arbitrary pore geometry can adequately be modeled using the Temperature Expansion Model. ${ }^{1,2}$ This model is based on the analogy of swelling with thermal expansion. ${ }^{6}$ It allows easy implementation into commercial finite element tools like Abaqus, which is used in the current studies. 


\subsection{Flow and filtration}

Based on the simulations of the pore opening, the microfluidics of the system can be described. We suppose a Stokes flow $(\mathrm{Re} \ll 1)$ which is true for microfluidic systems. ${ }^{14}$ In our previous work, ${ }^{3}$ we have derived that the flow profiles of free and blocked pores can be described using the Poisson's equation of Hagen-Poiseuille flow. From this, the flux decline under constant pressure regime can be derived when particles block a single pore. This characteristic flux drop $\Delta \dot{V}_{\text {char }}$ is used to derive the total number of blocked pores in a membrane with multiple pores. The influence of each pore is represented as a fluidic resistance inside a microfluidic equivalent circuit model, see section 2.4 .

\subsection{Measurement process}

There are different possibilities to use the characteristic flux drop $\Delta \dot{V}_{\text {char }}$ to develop measurement procedures. In the current work, we present the static measurement approach. The following steps are taken in order to obtain the size profile of particles in the flow:

0. Before starting the measurement process, the membrane is tuned to the smallest diameter $d_{\text {pore }, 1}$ that can be expected in the particle flow. Here, the tuning is done by changing the environmental temperature to $\vartheta=\vartheta_{1}$.

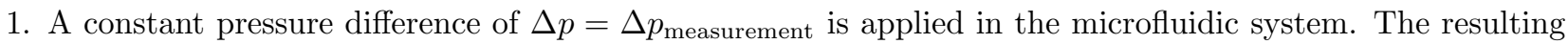
flow transports all particles of a sample to the membrane. All particles with diameter $d_{\text {particle }} \geq d_{\text {pore }, 1}$ lead to blocking of one pore each. The fluidic resistance of the blocked pores increases. From the resulting flow, the number of blocking particles $N_{\text {blockers, } 1}$ can be derived.

2. The temperature is changed to $\vartheta_{2}>\vartheta_{1}$ which results in pore opening to $d_{\text {pore }, 2}$. Now, particles with diameters $d_{\text {particle }}<d_{\text {pore }, 2}$ can pass through the membrane. From the new flux, the number of blockers $N_{\text {blocker,2 }}$ can be derived. The difference $N=N_{\text {blocker,1 }}-N_{\text {blocker,2 }}$ gives the number of particles in the respective particle class.

n. The process is continued for every particle class until there are no more blocking particles left.

The process scheme is depicted in figure 3a. In the current work a particle class is defined by the largest particle inside a specific range, e.g. $[10 \mu \mathrm{m} \ldots 20 \mu \mathrm{m}]$ all particles smaller than $20 \mu \mathrm{m}$ and larger than $10 \mu \mathrm{m}$. The size of particle classes depends on the accuracy of temperature control and indicates the measurement accuracy of the process.

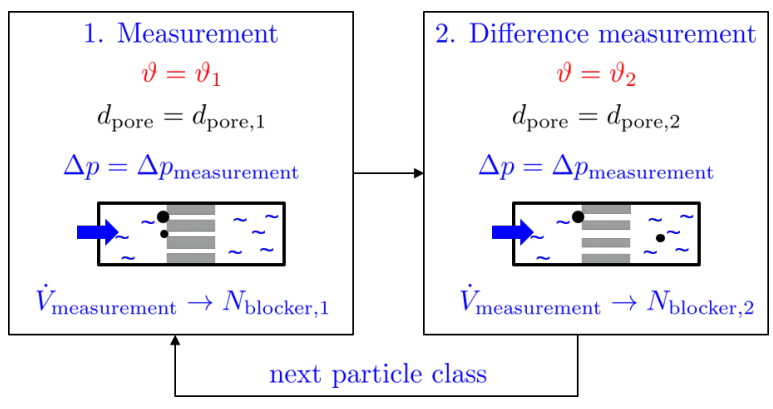

(a)

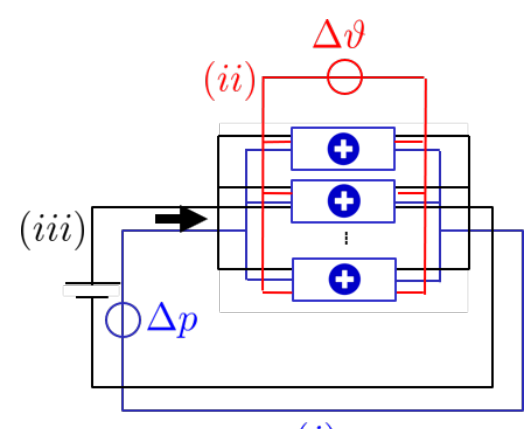

(i)

(b)

Figure 3: (a) Measurement process scheme which can be represented by a (b) circuit model consisting of a (i) fluidic, (ii) temperature and (iii) particle circuit. 


\subsection{Circuit model for the measurement process}

The measurement process described above can be described using an equivalent circuit description. ${ }^{15,16}$ The current model consists of three circuits and is depicted in Figure 3b:

(i). The fluidic circuit which represents the microfluidic flow of water through the system. The fluidic behavior is governed by the processes described in section 2.2 .

(ii). The temperature circuit which controls the fludic resistances of the opening pores in the network. This circuit represents the applied environmental temperature for opening and closing the hydrogel pores. The pore opening and closing behavior is described in section 2.1.

(iii). The circuit for particle transport and blocking which is controlled by cotransport with the first circuit.

The development of the fluidic resistance $R^{\text {fluidic }}$ over temperature that is generated by the pores is depicted for different pore geometries in free and blocked configuration in figure $4 \mathrm{a}$ and $4 \mathrm{~b}$ respectively. The values were derived from the applied pressure $\Delta p$ and resulting flow $\dot{V}$ from our previous work ${ }^{3}$ in analogy to OHM's law

$$
R^{\text {fluidic }}=\frac{\Delta p}{\dot{V}} .
$$

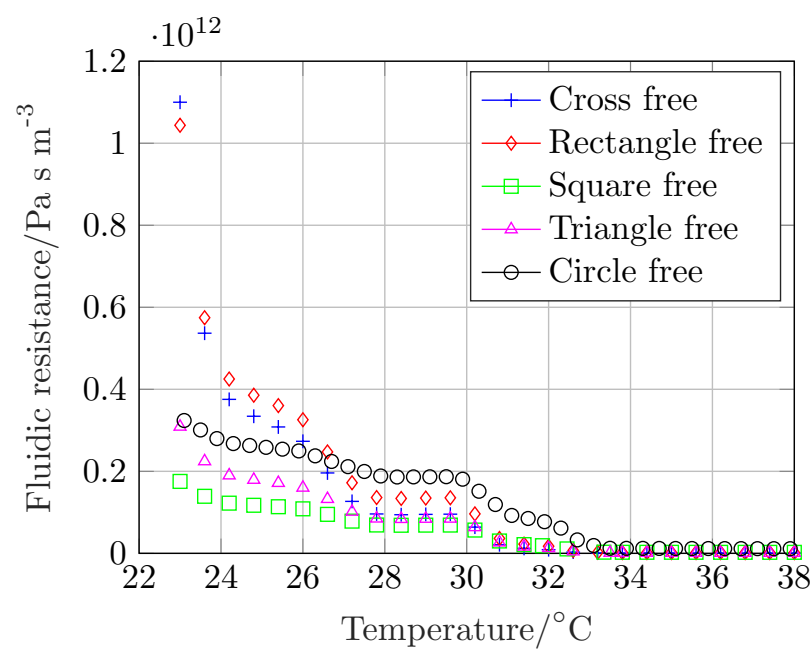

(a)

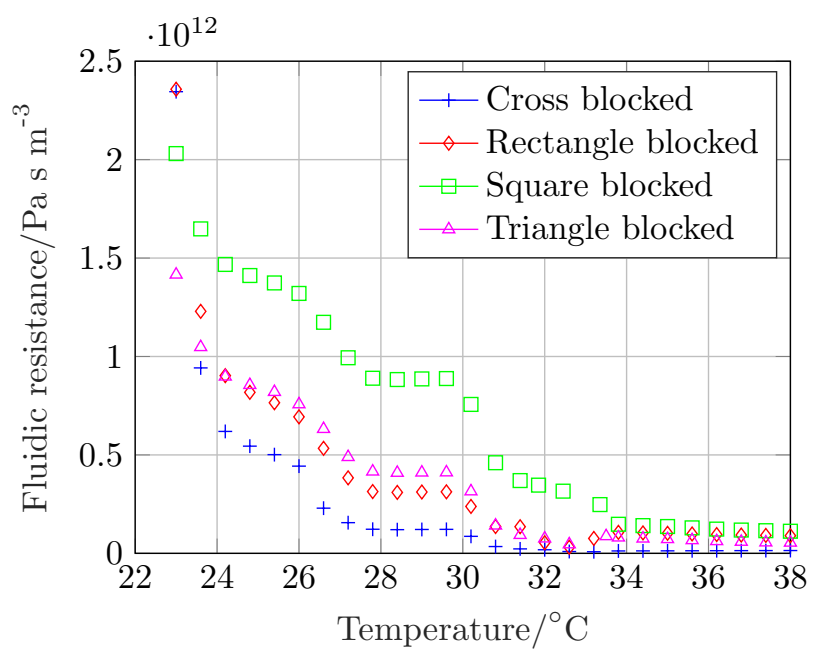

(b)

Figure 4: Fluidic resistances over temperature for different pore shapes in (a) free and (b) blocked state.

\section{MEASUREMENT PROCESS SIMULATION}

With the methods of modeling described in the previous section, a measurement process of the cell separation system can be simulated. In the current work, the actuation range of the measurement system is limited to a degree that particles inside the diameter range of $20 \mu \mathrm{m}$ (quasi-closed pores at $23^{\circ} \mathrm{C}$ ) up to $180 \mu \mathrm{m}$ (open pores at $38^{\circ} \mathrm{C}$ ) can be detected. ${ }^{1}$ Please note that future optimization of the membrane fabrication process makes it possible to bring it into the desired range of $7.5 \mu \mathrm{m} \pm 4 \mu \mathrm{m}$ for red blood cells.

For the current demonstration of the measurement method, we give a discrete particle size profile as a Gaussian distribution of $N=71$ particles with the peak at $100 \mu \mathrm{m}$, see Figure 5a. The goal of the measurement process is now to acquire the same distribution within the limits of its measurement accuracy.

In Figure 5b, the temperature steps for the process described in section 2.3 are depicted. Here, 10 classes were chosen so that the particle diameters are equally spaced. Due to the nonlinearity in the swelling curve of PNiPAAm, this leads to unequally spaced temperature steps. Near the reference temperature of $32{ }^{\circ} \mathrm{C}$, the 
swelling-stimulus curve is very steep, ${ }^{1}$ therefore the same diameter change has to be achieved with smaller temperature steps. The given temperatures in the measurement process define the respective particle classes. Due to the active behavior of the pores, they also define a range of particle sizes. The chosen classes are depicted in Table 1a.

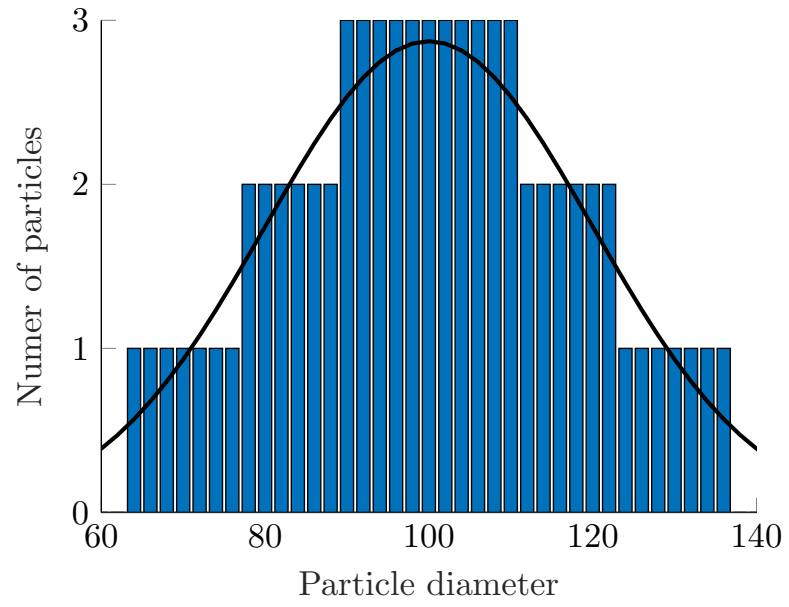

(a)

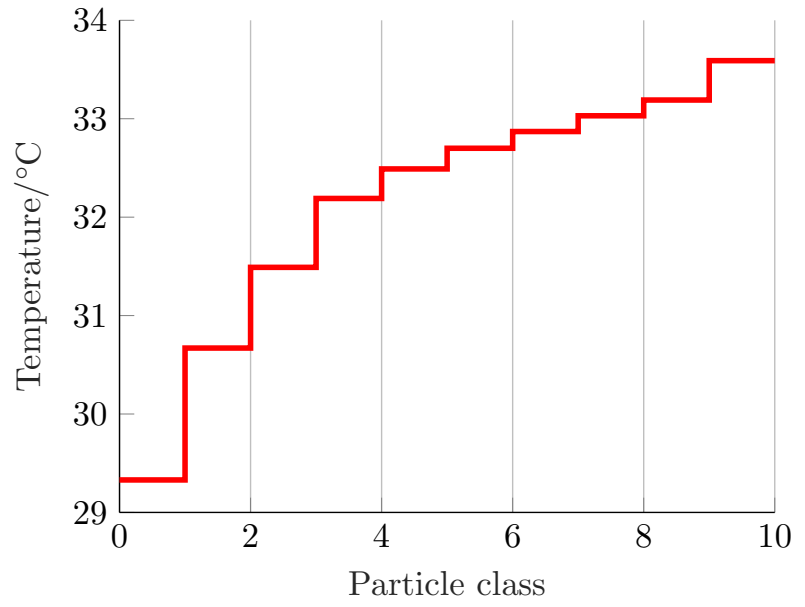

(b)

Figure 5: (a) Given particle profile of 71 particles in a Gaussian distribution for the performed studies and (b) subdivision into equally spaced particle classes due to temperature steps.

During the measurement process, the overall flow through the microfluidic membrane system for an applied pressure difference of $\Delta p_{\text {measurement }}=12 \mathrm{~Pa}$ is simulated. An active membrane with a total of 72 pores according to our previous works ${ }^{4}$ is regarded. In Figure 6 a, the resulting flux $\dot{V}_{\text {measurement }}$ for every temperature step is shown in the case that (i) all pores were free (upper curve) and that (ii) all pores were blocked (lower curve). For the particle-laden flow, partial blocking occurs, which leads to the intermediate curve. From the ratio between maximum, minimum and actual flux, the number of blocked pores can be derived. For the given particle profile, this number of blocked pores is depicted in Figure $6 \mathrm{~b}$.

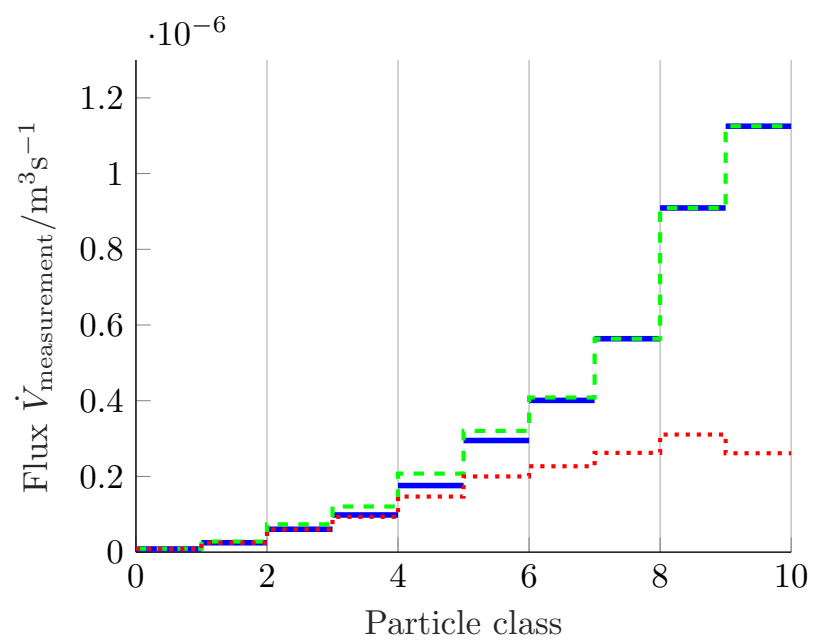

(a)

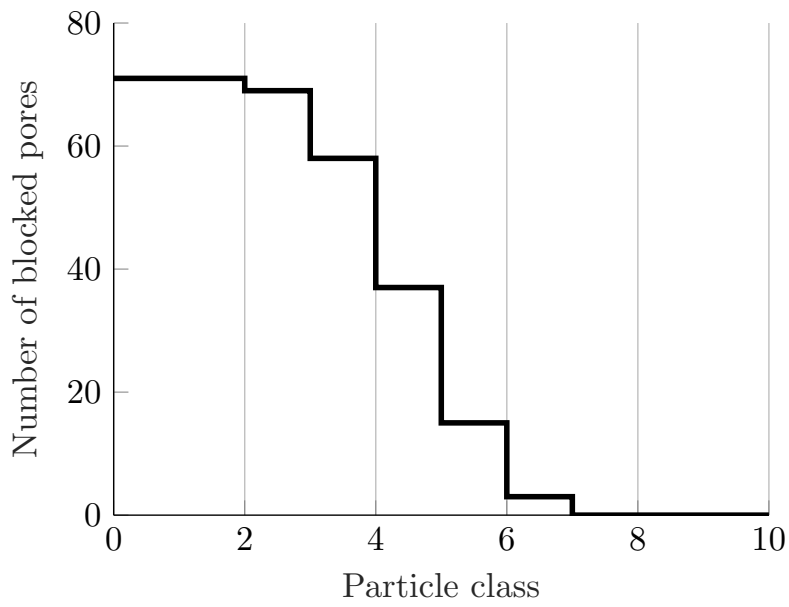

(b)

Figure 6: (a) The measurement flux $\dot{V}_{\text {measurement (partially blocked, solid black line) lies in between the maximum }}$ flux (all pores free, green dashed line) and the minimum flux (all pores blocked, red dotted line). (b) The number of blocked pores can directly be derived from the relation between actual, minimum and maximum flow. 
From these results, the number of particles per class can be derived, respectively. The reconstructed profile is depicted in Figure 7 and comprises all 71 particles. The difference of shape in respect to Figure 5a is due to the system's accuracy: For larger temperature steps more particles fall into the defined class. The difference in shape diminishes with more classes.

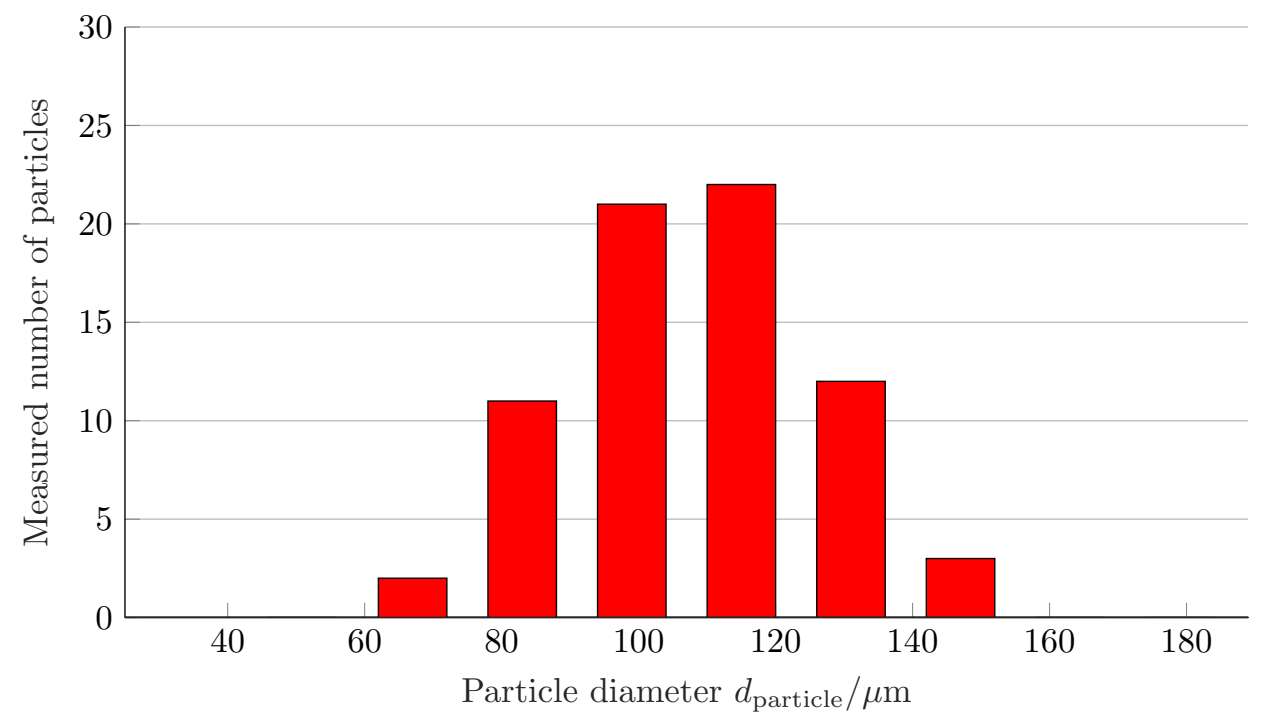

Figure 7: Derived number of particles versus particle diameter.

The number of particles in each particle class in the reconstructed profile respects the total amount of particles from the given profile in Figure 5a. The difference in shape is due to the number of classes and converges towards the original profile with more classes. The counted particles for the current 10 classes are also depicted in Table 1b. The number of counted particles in each class is derived from the measured pressure loss.

Table 1: (a) Temperature ranges and particle sizes in the respective particle classes (b) Number of counted particles.

(b)

\begin{tabular}{|c|c|c|}
\hline Class & Temperature & Particle range \\
\hline 1 & $\vartheta_{1}=30.05^{\circ} \mathrm{C}$ & {$[0 \mu \mathrm{m} \ldots 35 \mu \mathrm{m}]$} \\
\hline 2 & $\vartheta_{2}=30.67^{\circ} \mathrm{C}$ & {$[35 \mu \mathrm{m} \ldots 51 \mu \mathrm{m}]$} \\
\hline 3 & $\vartheta_{3}=31.45^{\circ} \mathrm{C}$ & {$[51 \mu \mathrm{m} \ldots 67 \mu \mathrm{m}]$} \\
\hline 4 & $\vartheta_{4}=32.28^{\circ} \mathrm{C}$ & {$[67 \mu \mathrm{m} \ldots 83 \mu \mathrm{m}]$} \\
\hline 5 & $\vartheta_{5}=32.52^{\circ} \mathrm{C}$ & {$[83 \mu \mathrm{m} \ldots 99 \mu \mathrm{m}]$} \\
\hline 6 & $\vartheta_{6}=32.69^{\circ} \mathrm{C}$ & {$[99 \mu \mathrm{m} \ldots 115 \mu \mathrm{m}]$} \\
\hline 7 & $\vartheta_{7}=32.85^{\circ} \mathrm{C}$ & {$[115 \mu \mathrm{m} \ldots 131 \mu \mathrm{m}]$} \\
\hline 8 & $\vartheta_{8}=33.01^{\circ} \mathrm{C}$ & {$[131 \mu \mathrm{m} \ldots 147 \mu \mathrm{m}]$} \\
\hline 9 & $\vartheta_{9}=33.18^{\circ} \mathrm{C}$ & {$[147 \mu \mathrm{m} \ldots 163 \mu \mathrm{m}]$} \\
\hline 10 & $\vartheta_{10}=33.34^{\circ} \mathrm{C}$ & {$[163 \mu \mathrm{m} \ldots 179 \mu \mathrm{m}]$} \\
\hline
\end{tabular}

\begin{tabular}{|c|c|}
\hline Class & Particles \\
\hline 1 & 0 \\
\hline 2 & 0 \\
\hline 3 & 2 \\
\hline 4 & 11 \\
\hline 5 & 21 \\
\hline 6 & 22 \\
\hline 7 & 12 \\
\hline 8 & 3 \\
\hline 9 & 0 \\
\hline 10 & 0 \\
\hline
\end{tabular}




\section{CONCLUSION AND OUTLOOK}

In the current work, we have shown that an active membrane system based on switchable hydrogel pores is capable of acting as a measurement device for particle profiles. For this system, we derived a measurement process which is based on the step-wise opening of the active pores inside the membrane.

In order to show how measurement data of the current system can be handled, we simulated a measurement process with a given particle profile. From the previously derived change in flux, the number of particles in a particle range - which is defined by the pore opening - was derived. This was then used to reconstruct the particle profile of the given sample.

However, a very high number of pores is needed in the membrane in order to allow the application of this process. In future investigations, we will discuss different measurement processes which are based on the timedependent change of the pore diameters.

Further improvement of the membrane system's accuracy and miniaturization will allow the utilization for cell size profiles in nanoliter-sized blood samples. This leads to an autonomous microfluidic measurement system for blood parameters that can e.g. detect anemia or tumor metastasis.

\section{ACKNOWLEDGMENTS}

The present work is supported by the Deutsche Forschungsgemeinschaft (DFG) in the framework of the research training group "Hydrogel-based microsystems" DFG-GRK 1865 at Technische Universität Dresden.

\section{REFERENCES}

[1] Ehrenhofer, A., Bingel, G., Paschew, G., Tietze, M., Schröder, R., Richter, A., and Wallmersperger, T., "Permeation control in hydrogel-layered patterned pet membranes with defined switchable pore geometry experiments and numerical simulation," Sensors and Actuators B: Chemical 232, 499-505 (2016).

[2] Ehrenhofer, A., Wallmersperger, T., and Richter, A., "Simulation of controllable permeation in pnipaam coated membranes," in [Proc. SPIE 9800, Behavior and Mechanics of Multifunctional Materials and Composites 2016], Proc. SPIE 980016, 1-13 (2016).

[3] Ehrenhofer, A. and Wallmersperger, T., "Adjustable fluid and particle permeation through hydrogel composite membranes," Journal of Intelligent Material Systems and Structures 29 (3), 310-322 (2018).

[4] Ehrenhofer, A., Hahn, M., Hofmann, M., and Wallmersperger, T., "Mechanical behavior and pore integration density optimization of switchable hydrogel composite membranes," submitted to Journal of Intelligent Material Systems and Structures (2019).

[5] Gerlach, G. and Arndt, K.-F., [Hydrogel Sensors and Actuators: Engineering and Technology], vol. 6, Springer (2009).

[6] Ehrenhofer, A., Elstner, M., and Wallmersperger, T., "Normalization of hydrogel swelling behavior for sensoric and actuatoric applications," Sensors and Actuators B: Chemical 255, 1343 - 1353 (2018).

[7] Leichsenring, P. and Wallmersperger, T., "Modeling and simulation of the chemically induced swelling behavior of anionic polyelectrolyte gels by applying the theory of porous media," Smart Materials and Structures 26(3), 1-15 (2017).

[8] Acartürk, A. Y., Simulation of charged hydrated porous materials, PhD thesis, University of Stuttgart, (2009).

[9] Ehlers, W., "Grundlegende Konzepte in der Theorie Poröser Medien," Technische Mechanik 16, 63-76 (1996).

[10] Sobczyk, M. and Wallmersperger, T., "Modeling and simulation of the electro-chemical behavior of chemically stimulated polyelectrolyte hydrogel layer composites," Journal of Intelligent Material Systems and Structures 27(13), 1725-1737 (2016).

[11] Shields, C. W., Reyes, C. D., and López, G. P., "Microfluidic cell sorting: A review of the advances in the separation of cells from debulking to rare cell isolation," Lab on a Chip 15(5), 1230-1249 (2015). 
[12] Guck, J., Schinkinger, S., Lincoln, B., Wottawah, F., Ebert, S., Romeyke, M., Lenz, D., Erickson, H. M., Ananthakrishnan, R., Mitchell, D., Käs, J., Ulvick, S., and Bilby, C., "Optical deformability as an inherent cell marker for testing malignant transformation and metastatic competence," Biophysical Journal 88(5), $3689-3698$ (2005).

[13] Adrus, N. and Ulbricht, M., "Novel hydrogel pore-filled composite membranes with tunable and temperature-responsive size-selectivity," J. Mater. Chem. 22, 3088-3098 (2012).

[14] Whitesides, G. M., "The origins and the future of microfluidics," Nature 442(7101), 368-373 (2006).

[15] Chávez, J. P., Voigt, A., Schreiter, J., Marschner, U., Siegmund, S., and Richter, A., "A new self-excited chemo-fluidic oscillator based on stimuli-responsive hydrogels: mathematical modeling and dynamic behavior," Applied Mathematical Modelling 40, 9719-9738 (2016).

[16] Paschew, G., Schreiter, J., Voigt, A., Pini, C., Chávez, J. P., Allerdißen, M., Marschner, U., Siegmund, S., Schüffny, R., Jülicher, F., Richter, A., "Autonomous chemical oscillator circuit based on bidirectional chemical-microfluidic coupling," Advanced Materials Technologies 1, 1-6 (2016). 